\title{
Fatores Associados com a Ocorrência de Hipoxemia no Período Pós-Anestésico Imediato *
}

\section{Factors Associated to Hypoxemia in the Immediate Postoperative Period}

Getúlio Rodrigues de Oliveira Filho, TSA ${ }^{1}$, Jorge Hamilton Soares Garcia, TSA ${ }^{3}$, Márcia Regina Ghellar, TSA ${ }^{2}$, Marcos Antônio Nicolodi, TSA ${ }^{3}$, Asmir Luiz Boso, TSA ${ }^{2}$, Adilson José Dal Mago, TSA ${ }^{3}$

\section{RESUMO}

Oliveira Filho GR, Garcia JHS, Ghellar MR, Nicolodi MA, Boso AL, Dal Mago AJ - Fatores Associados com a Ocorrência de Hipoxemia no Período Pós-Anestésico Imediato

Justificativa e Objetivos - A hipoxemia pós-anestésica é um evento respiratório crítico que aumenta a morbidade pós-operatória. Este estudo visou identificar fatores relacionados à ocorrência de hipoxemia no período pós-operatório imediato.

Método - Foram incluídos 204 pacientes admitidos à sala de recuperação pós-anestésica respirando ar ambiente. A saturação periférica da oxi-hemoglobina $\left(\mathrm{SpO}_{2}\right)$, as pressões arteriais sistólica (PAS) e diastólica (PAD) e a freqüência cardíaca (FC) foram medidas a intervalos de 5 minutos desde a admissão do paciente até 20 minutos após. Escores de sedação, de dor e de adequação da ventilação foram atribuídos nos mesmos momentos. $\mathrm{SpO}_{2}$ menor que $92 \%$ foi definida como hipoxemia e indicação para oxigenioterapia. Foram registrados: idade, sexo, peso, altura, história de fumo, de $D P O C$ e de diabete melito, $\mathrm{SpO}_{2}$ na chegada à sala de cirurgia, tipo de anestesia, região operada, duração da anestesia, uso de opióides neuro-axiais e drogas utilizadas no período peri-operatório e respectivas doses. A associação destes fatores com hipoxemia foi definida por regressão logística.

Resultados - Quarenta e nove pacientes (24,01\%) apresentaram $\mathrm{SpO}_{2}$ menor que $92 \%$ durante o período de observação. Foram indicadores de hipoxemia [relação de chances (limites de $95 \%$ de confiança)]: idade maior que 55 anos $[4,32(1,70 ; 10,95)], \mathrm{SpO}_{2}$ pré-operatória menor que $95 \%$ $[7,47(1,50 ; 37,11)]$, anestesia geral com enflurano $[14,53$ $(2,54 ; 82,93)]$, hipoventilação detectada clinicamente $[34,82$ $(11,46 ; 105,84)]$. A PAS e a FC foram significativamente mais elevadas nos pacientes hipoxêmicos.

Conclusões - Existem fatores significativamente associados à ocorrência de hipoxemia pós-operatória, enquanto o uso do oxímetro de pulso permite a utilização seletiva de oxigenioterapia no período pós-anestésico imediato.

UNITERMOS - COMPLICAÇÕES: hipoxemia

\footnotetext{
* Recebido do (Received from) Hospital Governador Celso Ramos, CET/SBA Integrado de Anestesiologia da SES-SC - Florianópolis, SC

1. Responsável pelo CET/SBA

2. Instrutor do CET/SBA

3. Anestesiologista do Hospital Governador Celso Ramos
}

Apresentado (Submitted) em 12 de julho de 2000

Aceito (Accepted) para publicação em 17 de outubro de 2000

Correspondência para (Mail to):

Dr. Getúlio Rodrigues de Oliveira Filho

Rua José Cãndido Silva 179/402

88075-250 - Florianópolis, SC

E-mail: grof@th.com.br

(C) Sociedade Brasileira de Anestesiologia, 2001

\section{SUMMARY}

Oliveira Filho GR, Garcia JHS, Ghellar MR, Nicolodi MA, Boso AL, Dal Mago AJ - Factors Associated to Hypoxemia in the Immediate Postoperative Period

Background and Objectives - Postanesthetic hypoxemia is a critical respiratory event which increases postoperative morbidity. This study aimed to identify hypoxemia-related factors in the immediate postoperative period.

Methods - Participated in this study 204 patients admitted to the recovery room while breathing room air. Peripheral oxy-hemoglobin saturation $\left(\mathrm{SpO}_{2}\right)$, systolic $(\mathrm{SBP})$ and diastolic $(\mathrm{DBP})$ blood pressure, and heart rate (HR) were measured at 5-minute intervals since admission to the recovery room until 20 minutes later. Sedation, pain and ventilation adequacy scores were attributed to patients at the same moments. Hypoxemia was defined as a $\mathrm{SpO}_{2}$ below $92 \%$ and recommended oxygen therapy. The following data were recorded for each patient: age, gender, weight, height, smoking, history of diabetes and chronic obstructive pulmonary disease (COPD), preanesthetic $\mathrm{SpO}_{2}$ at arrival in the operating room, type and duration of anesthesia, surgery site, use of neuro-axial opioids and perioperative drugs. The association of those factors to postoperative hypoxemia was defined by logistic regression.

Results - Forty-nine patients (24.01\%) had $\mathrm{SpO}_{2}$ below $92 \%$ during the observation period. The following factors [odds ratio (95\% confidence limits)] were considered hypoxemia predictors: more than 55 years old [4.32 (1.70; 10.95)], preanesthetic $\mathrm{SpO}_{2}$ lower than $95 \%[7.47(1.50 ; 37.11)]$, general anesthesia with enflurane [14.53 (2.54; 82.93)], and clinically detectable hypoventilation [34.82 (11.46; 105.84)]. SBP and HR were significantly higher in hypoxemic patients.

Conclusions - There are factors closely related to the incidence of postoperative hypoxemia and the use of pulse oximetry allows for selective oxygen therapy during the immediate postanesthetic period.

KEY WORDS - COMPLICATIONS: hypoxemia

\section{INTRODUÇÃO} administração de oxigênio como rotina, durante o perío-
do pós-operatório imediato, fundamenta-se em estudos
que demonstraram alta prevalência de hipoxemia neste pe-
ríodo ${ }^{1,2}$ ou que notaram menor prevalência de hipoxemia
entre pacientes que receberam oxigênio ${ }^{3,4}$. Entretanto,
existem evidências de que a administração de oxigênio não
é necessária em até $63 \%$ dos pacientes durante o período
pós-operatório imediato ${ }^{5,6}$.
A hipoxemia ocorre a despeito de administração de oxigê-
nio ${ }^{2,7,8}$ e episódios hipoxêmicos não são diagnosticados na
maioria das vezes, pelo pessoal da Sala de Recuperação
Pós-Anestésica (SRPA) ${ }^{2}$. Entretanto, a utilização de oxíme- 
tro de pulso na SRPA permite identificar pacientes hipoxêmicos e indicar oxigenioterapia de forma seletiva ${ }^{5,9}$.

Diversos fatores dependentes do paciente, da técnica anestésica e da cirurgia associam-se com maiores chances de hipoxemia no período pós-operatório imediato ${ }^{7,8}$. Estes fatores podem facilitar a identificação de pacientes com maior probabilidade de desenvolver hipoxemia, que deverão ser, portanto, alvo de maior atenção do pessoal da SRPAe candidatos ao uso profilático e seletivo de oxigenioterapia.

Assim, este estudo teve como objetivo detectar os fatores dependentes dos pacientes, das cirurgias e das anestesias associados à ocorrência de hipoxemia no período pós-anestésico imediato.

\section{MÉTODO}

Com a aprovação da Comissão de Ética Médica do Hospital Governador Celso Ramos, foram incluídos 216 pacientes com idades entre 15 e 87 anos, de ambos os sexos, submetidos a cirurgias eletivas sob anestesia geral ou regional, admitidos seqüencialmente à SRPA. Foram excluídos os pacientes que estivessem sob oxigenioterapia ou ventilação mecânica. Imediatamente após a admissão, os pacientes foram monitorizados com oxímetro de pulso e monitor não invasivo, oscilométrico, de pressão arterial. A saturação periférica da oxi-hemoglobina $\left(\mathrm{SpO}_{2}\right)$, a freqüência cardíaca $(\mathrm{FC})$, as pressões arteriais sistólica (PAS) e diastólica (PAD) foram registradas durante a admissão (M0) e a intervalos de $5 \mathrm{mi}-$ nutos (M5, M10, M15) até o vigésimo minuto (M20). Nos mesmos momentos, foi atribuído a cada paciente um escore de sedação ( 0 = agitado, 1 = calmo, alerta, 2 = sonolento, desperta a comando verbal, 3 = sonolento, desperta à estimulação tátil e 4 = comatoso), um escore de adequação da respiração ( 0 = apnéia, 1 = incursões respiratórias limitadas, obstrução respiratória, broncoespasmo ou FR menor que 8 irpm e 2 = respiração livre), um escore de força muscular (1 = mantém aperto de mão por tempo $\geq 15$ segundos ou 0 = não mantém aperto de mão ou o faz por menos de 15 segundos) e um escore verbal de intensidade dolorosa ( $0=$ sem dor ou não responde, 1 = dor leve, 2 = dor moderada e 3 = dor forte). Durante o período de estudo, os pacientes somente receberam oxigênio, por cateter nasal $\left(3\right.$ L. min $\left.^{-1}\right)$, caso tenham apresentado $\mathrm{SpO}_{2}$ menor que $92 \%$. Foram coletados os seguintes dados: idade, sexo, peso, altura, história de fumo, doença pulmonar obstrutiva crônica (DPOC) ou diabete melito, $\mathrm{SpO}_{2}$ na chegada à sala de cirurgia, tipo de anestesia, região operada, duração da anestesia, uso neuro-axial de opióides e drogas utilizadas no período peri-operatório, com as respectivas doses. Hipoventilação detectada clinicamente foi definida como um escore de adequação da respiração igual a 1. Os pacientes foram divididos em hipoxêmicos, caso tenham apresentado, em qualquer momento do período de observação, $\mathrm{SpO}_{2}$ menor que $92 \%$ por mais de 30 segundos e em normoxêmicos, caso não tenham apresentado $\mathrm{SpO}_{2}$ menor que $92 \%$, sem oxigenioterapia, durante todo o período de estudo.
Os dados demográficos, a distribuição dos pacientes segundo a técnica anestésica, a região operada, a história de diabete melito, de DPOC e de tabagismo, o estado físico, os escores de adequação da ventilação, de sedação, de dor e de força muscular, a ocorrência de hipoventilação, a freqüência de $\mathrm{SpO}_{2}$ menor que $95 \%$ na chegada à sala de cirurgia, a idade maior que 55 anos e a freqüência da utilização de cada fármaco no período peri-operatório foram comparados, entre os pacientes que apresentaram e os que não apresentaram hipoxemia, pelos testes $t$ de Student para amostras independentes (as variáveis contínuas) ou qui-quadrado (as variáveis categóricas), a partir do qual foram calculadas as respectivas relações de chances e seus limites de $95 \%$ de confiança. As variáveis que diferiram significativamente entre os subgrupos pelo teste $t$ de Student ou que apresentaram associação significativa com hipoxemia pelo teste do qui-quadrado, foram utilizadas como variáveis independentes em análise de regressão logística retrógrada, na qual a presença ou não de hipoxemia $\left(\mathrm{SpO}_{2}\right.$ menor que $\left.92 \%\right)$ foi a variável dependente.

Os dados hemodinâmicos foram comparados, entre os pacientes hipoxêmicos e os normoxêmicos, por análise de co-variância bi-fatorial para medidas repetidas, sendo a co-variável, a $\mathrm{SpO}_{2}$ em cada momento do estudo, seguida pelo teste post hoc de Spjotvoll-Stolline.

$\mathrm{A} \mathrm{SpO}_{2}$ foi comparada pelos testes de Friedman, Wilcoxon pareado (comparações intra-grupos) e Mann-Whitney (comparações momento a momento, entre os grupos).

O nível de significância aceito foi de $5 \%$.

\section{RESULTADOS}

Foram excluídos da análise 12 pacientes que receberam oxigenioterapia na SRPA por motivos outros que o previsto no protocolo deste estudo, como anemia (2 pacientes), calafrios (2 pacientes), broncoespasmo (1 paciente), hipotensão arterial (4 pacientes) e motivo desconhecido (2 pacientes).

As características demográficas dos 204 pacientes cujos dados foram analisados estão resumidas na tabela I. Destes, 49 $(23,14 \%)$ apresentaram $\mathrm{SpO}_{2}$ menor que $92 \%$ por mais de 30 segundos, durante o período de observação, e receberam oxigenioterapia. Os pacientes hipoxêmicos apresentaram idade significativamente maior do que os normoxêmicos (Tabela II).

Tabela I - Dados Demográficos e Principais Características da Amostra

\begin{tabular}{lc}
\hline${\text { Idade }(\text { anos })^{1}}^{1}$ & $45,96 \pm 17,91$ \\
${\text { Sexo }(\mathrm{M} / \mathrm{F})^{2}}^{2}$ & $118 / 86$ \\
Peso $(\mathrm{kg})^{1}$ & $69,67 \pm 11,80$ \\
Altura $(\mathrm{m})^{1}$ & $1,66 \pm 0,09$ \\
$\mathrm{ASA}(\mathrm{I}-\mathrm{II}-\mathrm{III}-\mathrm{IV})^{2}$ & $83 / 100 / 20 / 1$ \\
Fumo (presente/ausente) ${ }^{2}$ & $61 / 143$ \\
DPOC (presente/ausente) $^{2}$ & $5 / 199$ \\
Diabete (presente/ausente) $^{2}$ & $11 / 193$ \\
Anestesia geral/regional $^{2}$ & $101 / 103$ \\
Cirurgia superficial/abdominal $^{2}$ & $74 / 130$ \\
\hline
\end{tabular}

$1=$ Valores expressos como Média \pm DP

$2=$ Valores expressos como freqüências 
Tabela II - Dados Demográficos - Comparação entre os Pacientes Hipoxêmicos e os Normoxêmicos

\begin{tabular}{lcc}
\hline & Normoxêmicos & Hipoxêmicos \\
\hline Idade $\left(\right.$ anos) $^{1}$ & $42,99 \pm 15.78$ & $55,36 \pm 18,30$ * \\
Sexo $(\mathrm{M} / \mathrm{F})^{2}$ & $93 / 92$ & $25 / 24$ \\
Peso $(\mathrm{kg})^{1}$ & $68,86 \pm 11,28$ & $72,25 \pm 13,08$ \\
Altura $(\mathrm{m})^{1}$ & $1,66 \pm 0,09$ & $1,67 \pm 0,09$ \\
\hline
\end{tabular}

1 = Valores expressos como Média $\pm \mathrm{DP}$

$2=$ Valores representam o número de pacientes em cada categoria

${ }^{*}=p<0,05$ entre os grupos

As seguintes variáveis obtiveram significância estatística na análise individual pelo teste do qui-quadrado: o estado físico (ASA) III, a idade maior que 55 anos, a $\mathrm{SpO}_{2}$ pré-operatória menor que $95 \%$, a história de DPOC e de diabete, a sedação profunda (paciente desperta somente ao toque ou não desperta), a força muscular inadequada (paciente não consegue manter aperto de mão por 15 segundos), a hipoventilação detectada clinicamente, o uso de diazepam e das associações de fentanil com diazepam ou com midazolam para sedação durante anestesias regionais, a associação de fentanil com morfina, o uso do vecurônio, do enflurano, do isoflurano e do $\mathrm{N}_{2} \mathrm{O}$, a anestesia geral e a anestesia regional (Tabelas III e IV). O modelo de regressão logística que melhor se adaptou à amostra incluiu anestesia geral com enflurano, hipoventilação detectada clinicamente, idade acima de 55 anos e a $\mathrm{SpO}_{2}$ de chegada na sala de cirurgia menor que $95 \%$ (Tabela V). Com este modelo, $96,12 \%$ dos pacientes normoxêmicos e $65,30 \%$ dos hipoxêmicos foram corretamente classificados, utilizando-se o limite de 0,5 para o logit.

Tabela V - Variáveis Significativamente Associadas à Hipoxemia Pós-Anestésica, Segundo o Modelo de Regressão Logística

\begin{tabular}{lc}
\hline Variável & $\begin{array}{c}\text { Relação de chances e } \\
\text { limites de confiança }\end{array}$ \\
\hline Idade > 55 anos & $4,32(1,70 ; 10,95)$ \\
$\mathrm{SpO}_{2}$ pré-operatória $<95 \%$ & $7,47(1,50 ; 37,11)$ \\
Anestesia geral com enflurano & $14,53(2,54 ; 82,93)$ \\
Hipoventilação detectada clinicamente & $34,82(11,46 ; 105,84)$ \\
\hline
\end{tabular}

Não atingiram significância estatística em nenhuma das análises: o peso, o sexo, o índice de massa corporal, o estado físico, a história de fumo, o volume de cristalóides administrados durante a cirurgia, a duração e o local da cirurgia, os escores de dor, o uso de tiopental, de propofol, de midazolam, de fentanil, de alfentanil, de morfina, de atracúrio, de sevoflurano ou de opióides neuro-axiais (Tabela VI).

Tabela III - Variáveis Significativamente Associadas à Hipoxemia, sua Prevalência em Pacientes Normoxêmicos e Hipoxêmicos e Respectivas Relações de Chances baseadas na Análise de Tabelas de Contingência (Qui-Quadrado)

\begin{tabular}{|c|c|c|c|}
\hline & Normoxêmicos & Hipoxêmicos & $\begin{array}{l}\text { Relação de chances e respectivos } \\
\text { limites de } 95 \% \text { de confiança }\end{array}$ \\
\hline Estado físico (ASA) III & 5,86 & 24,48 * & $5,95(2,07 ; 17,41)$ \\
\hline Idade $>55$ anos & 18,70 & 53,06 * & $4,91(2,32 ; 10,41)$ \\
\hline Diabete & 2,58 & 14,28 * & $6,29(1,55 ; 27,09)$ \\
\hline DPOC & 0,64 & 8,16 * & $13,68(1,38 ; 112,30)$ \\
\hline $\mathrm{SpO}_{2}$ pré-operatória $<95 \%$ & 2,58 & 18,36 * & $8,49(2,23 ; 34,84)$ \\
\hline Anestesia geral & 41,29 & 79,59 * & $5,54(2,44 ; 12,85)$ \\
\hline Anestesia regional & 20,4 & $58,7 *$ & $0,18(0,07 ; 0,4)$ \\
\hline Sedação profunda ${ }^{1}$ & 5,80 & 20,40 & $4,38(1,50 ; 12,82)$ \\
\hline Força muscular inadequada ${ }^{2}$ & 10,32 & 36,73 & $5,35(2,28 ; 12,62)$ \\
\hline Hipoventilação & 3,87 & 57,14 * & $31,11(11,30 ; 102,16)$ \\
\hline
\end{tabular}

1 = desperta somente ao toque ou não desperta

2 = não sustenta aperto de mão por 15 segundos

$*=p<0,05$ entre pacientes normoxêmicos e hipoxêmicos

Tabela IV - Drogas Utilizadas no Período Peri-Operatório Associadas à Hipoxemia, sua Prevalência de Uso (\%) em Pacientes Normoxêmicos e Hipoxêmicos e Respectivas Relações de Chances Baseadas na Análise de Tabelas de Contingência (Qui-Quadrado)

\begin{tabular}{|c|c|c|c|}
\hline & Normoxêmicos & Hipoxêmicos & $\begin{array}{c}\text { Relação de chances } \\
\text { (limites de } 95 \% \text { de confiança) }\end{array}$ \\
\hline Diazepam $^{1}$ & 16,77 & 4,08 * & $0,21(0,04 ; 0,96)$ \\
\hline Fentanil + diazepam ${ }^{1}$ & 13,54 & 2,04 * & $0,13(0,01 ; 0,97)$ \\
\hline Fentanil + morfina & 9,67 & 22,44 * & $2,7(1,05 ; 6,88)$ \\
\hline Vecurônio & 16,12 & 30,61 * & $2,29(1,02 ; 5,13)$ \\
\hline Enflurano & 1,93 & 14,28 * & $8,44(1,85 ; 32,38)$ \\
\hline $\mathrm{N}_{2} \mathrm{O}^{2}$ & 22,58 & 40,81 * & $3,05(1,46 ; 6,34)$ \\
\hline
\end{tabular}

$1=$ utilizados apenas para sedação durante anestesias regionais

2 = utilizado com anestésicos voláteis

${ }^{*} p<0,05$ entre os grupos

Revista Brasileira de Anestesiologia

Vol. 51, No 3, Maio - Junho, 2001 
Tabela VI - Fatores Não Associados Significativamente à Hipoxemia Pós-Anestésica

\begin{tabular}{|c|c|c|}
\hline Variável & Normoxêmicos & Hipoxêmicos \\
\hline Peso $(\mathrm{kg})^{1}$ & $68,86 \pm 11,28$ & $72,25 \pm 13,08$ \\
\hline $\operatorname{IMC}\left(\mathrm{kg} \cdot \mathrm{m}^{-2}\right)^{1}$ & $24,94 \pm 3,98$ & $25,86 \pm 5,02$ \\
\hline Cristalóides (L) ${ }^{1}$ & $1,45 \pm 0,67$ & $1,52 \pm 0,63$ \\
\hline Duração (min) ${ }^{1}$ & $105,77 \pm 47,21$ & $125 \pm 56,43$ * \\
\hline Cirurgias cavitárias ${ }^{2}$ & 32,25 & 48,97 \\
\hline Sevoflurano $^{2}$ & 2,58 & 10,20 \\
\hline Tiopental $^{2}$ & 21,93 & 30,61 \\
\hline Propofol $^{2}$ & 21,29 & 34,69 \\
\hline Midazolam ${ }^{2}$ & 35,48 & 10,20 \\
\hline Fentanil $^{2}$ & 68,38 & 69,38 \\
\hline Alfentanil $^{2}$ & 7,74 & 14,28 \\
\hline Morfina $^{2}$ & 14,83 & 28,57 \\
\hline Atracúrio $^{2}$ & 13,54 & 26,53 \\
\hline Opióide espinhal $^{2}$ & 10,96 & 18,36 \\
\hline
\end{tabular}

1 = Valores expressos como Média \pm DP

2 = Valores expressos como percentuais de ocorrência

${ }^{*} p<0,05$ entre os grupos

A pressão arterial sistólica, a freqüência cardíaca e a saturação periférica da hemoglobina estão apresentadas nas figuras 1,2 e 3 , respectivamente.

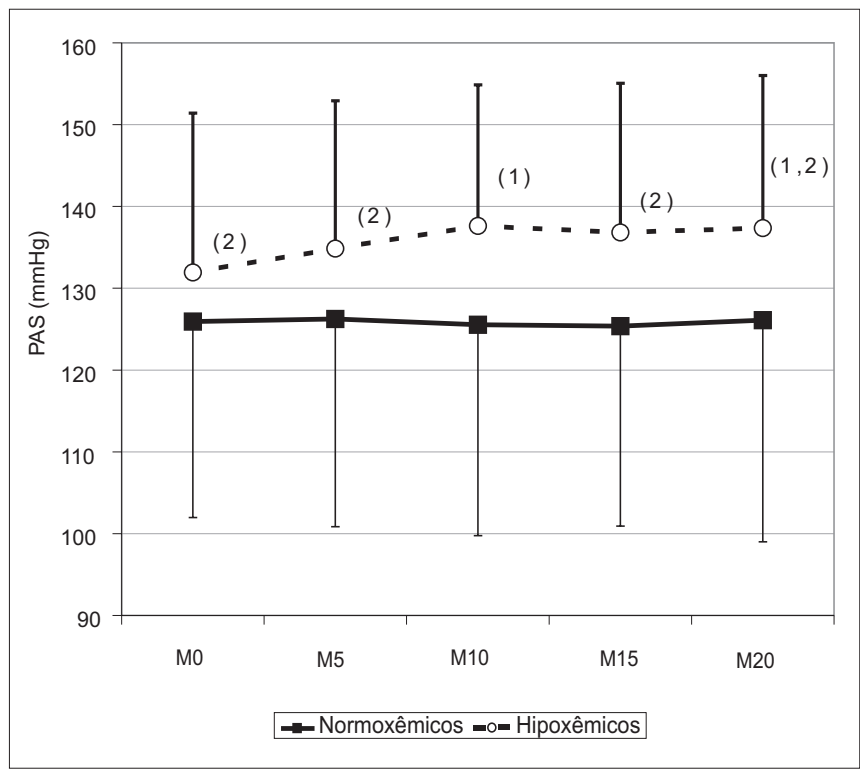

Figura 1 - Pressão Arterial Sistólica (Média \pm DP)

$1=p<0,05$ comparado com M0

$2=p<0,05$ entre os grupos

\section{DISCUSSÃO}

A hipoxemia pós-operatória tem como causas o controle inadequado da ventilação ou da patência das vias aéreas, resultante do efeito residual de anestésicos e/ou bloqueadores neuromusculares e a inadequação da relação ventilação/perfusão causada, principalmente, por zonas de atelec-

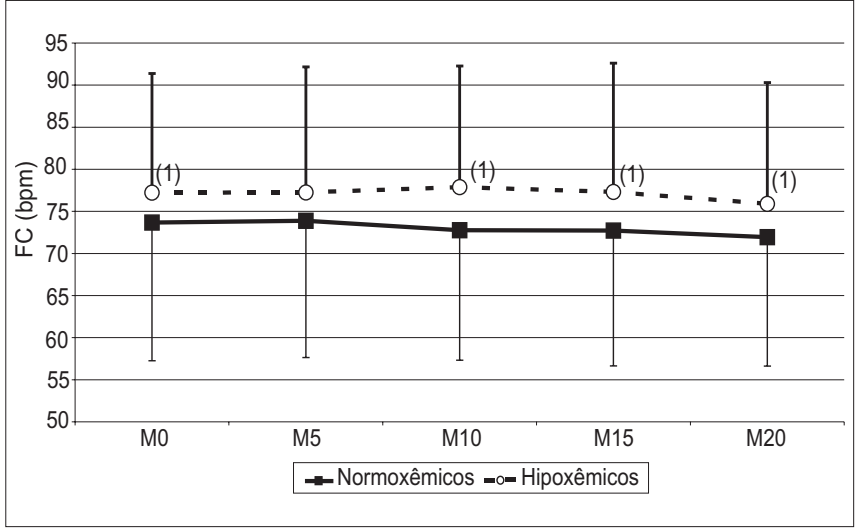

Figura 2 - Freqüência Cardíaca (Média \pm DP) $1=p<0,05$ entre os grupos

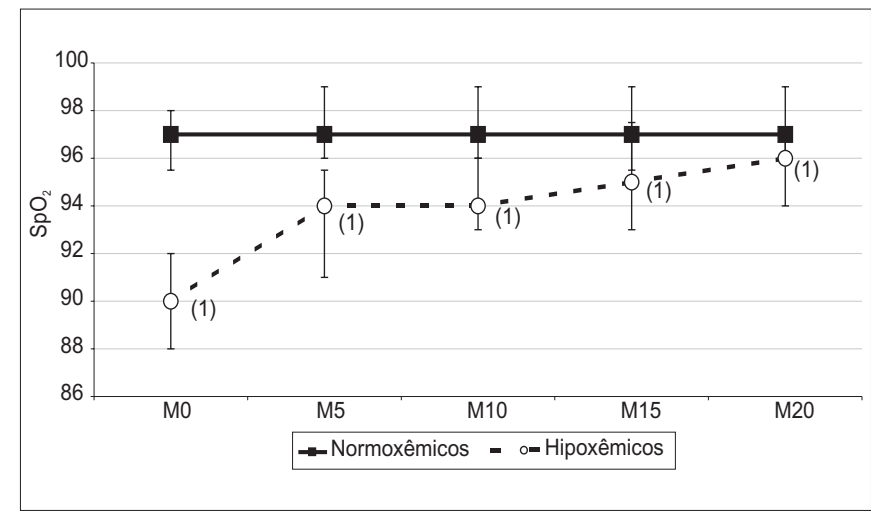

Figura 3 - Saturação Periférica da Hemoglobina (mediana, quartis inferior e superior)

$1=p<0,05$ comparado com M0

$p<0,05$ entre os grupos

tasia nas regiões dependentes dos pulmões ${ }^{10-12}$. Outras causas incluem estados de baixo débito cardíaco, anemia, calafrios termogênicos, dor e agitação ${ }^{13}$. A maior parte dos eventos hipoxêmicos ocorrem durante os 15 minutos subseqüentes à admissão dos pacientes na SRPA ${ }^{1,4}$.

A hipoxemia pós-anestésica associa-se à maior incidência de eventos cardiovasculares no período pós-operatório imediato, permanência mais longa de pacientes na SRPA e aumento do número de admissões não planejadas na UTI ${ }^{7}$. Aintrodução do oxímetro de pulso na monitorização de pacientes durante o período pós-anestésico imediato contribuiu para o aumento da identificação de episódios hipoxêmicos, de eventos cardíacos, como bradicardia, diminuição da ocorrência de isquemia miocárdica, utilização de maiores fluxos de oxigênio e maior utilização de oxigenioterapia após a alta da SRPA, sem, entretanto modificar a taxa de complicações ou a mortalidade pós-operatória ${ }^{9}$.

Define-se hipoxemia como a saturação arterial da oxi-hemoglobina $\left(\mathrm{SaO}_{2}\right)$ inferior a $90 \%$, que corresponde a uma $\mathrm{PaO}_{2}$ aproximadamente igual a $60 \mathrm{mmHg}$. Esta saturação encontra-se na porção inclinada da curva de dissociação da hemo-

Revista Brasileira de Anestesiologia Vol. 51, Nº 3, Maio - Junho, 2001 
globina, implicando em que pequenas variações de $\mathrm{SaO}_{2}$ resultem em reduções acentuadas da $\mathrm{PaO}_{2}{ }^{13}$. Entretanto, a $\mathrm{SpO}_{2}$ superestima a $\mathrm{SaO}_{2}$ em 2 a $5 \%{ }^{14,15}$, justificando os limites inferiores de $\mathrm{SpO}_{2}$, entre 92 e $96 \%$, utilizados em diversos estudos $5,8,16,17$.

Nos estudos que utilizaram o limite de $\mathrm{SpO}_{2}$ em $90 \%$, hipoxemia ocorreu em $0,9 \%{ }^{7}, 4 \%{ }^{6}, 8,24 \%{ }^{18}, 20 \%{ }^{4}, 55 \%{ }^{2}$ e $80 \%{ }^{1}$ dos pacientes estudados. O limite de $\mathrm{SpO}_{2}$ em $94 \%$ associouse com prevalência de hipoxemia em $15 \%{ }^{17}$ e $37 \%{ }^{5}$ dos pacientes. Utilizando o mesmo limite de $\mathrm{SpO}_{2}$, pacientes que receberam anestesia geral e respiraram ar ambiente na SRPA tiveram prevalência maior $(44,7 \%)$ do que pacientes submetidos a anestesias regionais $(24 \%)$ ou que foram submetidos à anestesia geral e receberam oxigenioterapia pós-operatória $(26,1 \%)^{3}$. Nos estudos em que o limite da $\mathrm{SpO}_{2}$ foi de $92 \%$, hipoxemia ocorreu em $7 \%{ }^{16}$ e em $15 \%{ }^{8}$ dos pacientes, enquanto no presente estudo, hipoxemia ocorreu em $23,14 \%$ dos pacientes.

Em estudos precedentes, as variáveis associadas a maiores probabilidades de ocorrência de hipoxemia pós-anestésica imediata foram: sexo masculino, diabete melito, cirurgias de urgência, medicação pré-anestésica com opióides e sedativos, fentanil em doses acima de $2 \mu \mathrm{g} \cdot \mathrm{kg}^{-1} \cdot \mathrm{h}^{-1}$, associação de fentanil com morfina, tiopental comparado ao propofol $^{7}$, obesidade $^{1,7,8,18}$, idade acima de 60 anos ${ }^{2,7,8,17,18}$, duração da anestesia acima de 4 horas $^{2,7}$, infusão de mais de $1500 \mathrm{ml} \mathrm{de}$ cristalóides $^{8}$, estado físico (ASA) ${ }^{8}$ e anestesia geral ${ }^{8}$.

Neste estudo, a análise isolada das variáveis mostrou que se associaram significativamente com a ocorrência de hipoxemia no período pós-operatório imediato o estado físico ASA III, a idade acima de 55 anos, a história de DPOC ou de diabete melito, a $\mathrm{SpO}_{2}$ pré-operatória menor que $95 \%$, a anestesia geral, a associação de fentanil per-operatório com morfina pós-operatória, a utilização de vecurônio, de enflurano, de isoflurano e de $\mathrm{N}_{2} \mathrm{O}$, os estados de sedação pós-anestésica profunda, a incapacidade de manter um aperto de mão por 15 segundos e os sinais clínicos de ventilação inadequada.

Por outro lado, a anestesia regional, acompanhada ou não de sedação per-operatória com diazepam e associações de fentanil e diazepam ou midazolam, associou-se a chances significativamente menores de ocorrência de hipoxemia no período pós-anestésico imediato.

A análise isolada de variáveis tem o inconveniente de não levar em consideração o efeito de outros fatores que podem influenciar possíveis associações entre cada variável e um determinado evento. Por outro lado, a regressão logística tem porvantagem controlar todos os fatores incluídos no modelo, ao calcular a influência de cada uma das variáveis, independentemente, sobre a variável dependente dicótoma. Por esta razão, após a identificação das variáveis potencialmente associadas à ocorrência de hipoxemia pós-operatória, por análise isolada, foi aplicada a técnica de regressão logística, que apontou como indicadores independentes de hipoxemia pós-operatória apenas a idade maior que 55 anos, a $\mathrm{SpO}_{2}$ pré-operatória menor que $95 \%$, a anestesia geral com enflu-

Revista Brasileira de Anestesiologia

Vol. 51, № 3, Maio - Junho, 2001 rano e a hipoventilação detectada clinicamente. As demais variáveis, significativas nas análises isoladas, não adicionaram robustez ao modelo logístico, razão pela qual foram rejeitadas.

Pacientes idosos possuem diminuição da reserva respiratória, por fatores como a diminuição do controle nervoso da respiração, manifestado principalmente pelo aumento de episódios apnéicos durante o sono, alterações mecânicas da parede torácica (aumento da cifose dorsal, calcificação de cartilagens costais e diminuição da massa muscular respiratória) e alterações nos volumes e capacidades pulmonares (diminuição das capacidades respiratória máxima, pulmonar total e vital e aumento do volume de fechamento das vias aéreas) ${ }^{19}$. Além disto, os pacientes idosos são mais sensíveis aos efeitos depressores dos anestésicos inalatórios, sedativos e opióides. Por estas razões, estão mais expostos a hipoxemia pós-operatória, como demonstrado neste e em outros estudos ${ }^{2,7,8,16,18}$

Em voluntários sadios, $\mathrm{SpO}_{2}$ menor que $94 \%$ foi observada em apenas $13,3 \%{ }^{3}$. É provável que pacientes que apresentem $\mathrm{SpO}_{2}$ menor que $95 \%$ no período pré-operatório exibam alguma anormalidade da ventilação, seja hipoventilação causada, por exemplo, por medicação pré-anestésica ou anormalidades da relação ventilação/perfusão dos pulmões.

Pacientes submetidos à anestesia geral têm chances maiores de desenvolver hipoxemia pós-operatória ${ }^{3,8}$. Entretanto, neste estudo, somente a anestesia geral com enflurano associou-se significativamente à hipoxemia, no modelo logísti$\mathrm{co}$, embora a anestesia geral, o isoflurano e o $\mathrm{N}_{2} \mathrm{O}$ tenham se mostrado significativos nas análises isoladas. O enflurano é um potente depressor da ventilação e causa mais hipoxemia no período pós-operatório imediato e tardio ${ }^{20}$. Embora sua concentração no gás expirado não tenha sido medida, é possível que o efeito residual do enflurano possa contribuir para a ocorrência de hipoxemia no período pós-anestésico imediato.

A hipoventilação detectada clinicamente foi o fator mais fortemente associado à ocorrência de hipoxemia. Os parâmetros para definir hipoventilação foram a freqüência respiratória, a inspeção das incursões diafragmáticas e a detecção de anormalidades da ventilação, como a obstrução respiratória alta e o broncoespasmo. Desta forma, este resultado confirma o valor do exame clínico na detecção de fatores de risco para a ocorrência de hipoxemia pós-operatória precoce.

Assim como em outros estudos ${ }^{3,8}$, a anestesia regional associou-se a menores chances de ocorrência de hipoxemia pós-operatória, ainda que sob sedação com diazepam ou midazolam associados ou não ao fentanil.

Entre as variáveis estudadas, não obtiveram significância nem nas análises isoladas, nem na regressão logística o peso, o sexo, o índice de massa corporal, a história de tabagismo, o volume de cristalóides administrados durante a cirurgia, a duração e o local da cirurgia, o uso de tiopental, de propofol, de midazolam, de fentanil, de alfentanil, de morfina, de atracúrio, de sevoflurano ou de opióides neuro-axiais. Os 
pacientes hipoxêmicos não diferiram dos normoxêmicos quanto a estas variáveis, exceto pela duração da anestesia. Em estudo precedente ${ }^{2,7}$, a duração da anestesia somente se mostrou indicador de hipoxemia, quando superior a 4 horas. É possível que este estudo tenha falhado em identificar a duração dos procedimentos como fator significativamente associado com a ocorrência de hipoxemia pós-operatória pelo fato da duração média dos procedimentos ter sido inferior a 4 horas. $O$ tabagismo se mostrou indicador significativo de hipoxemia em um estudo ${ }^{2}$, enquanto em outros ${ }^{6,17}$, assim como neste, não obteve significância. Autilização de morfina para analgesia pós-operatória não se associou significativamente à ocorrência de hipoxemia repetindo o achado de outros autores ${ }^{7}$. Assim, o risco de hipoxemia não justifica limitar o uso de morfina para analgesia pós-operatória. As cirurgias cavitárias não apresentaram maior associação com hipoxemia do que as superficiais, concordando com os achados de outros autores ${ }^{2,7}$. Entretanto, em um estudo, as cirurgias cavitárias associaram-se a maiores percentuais de hipoxemia pós-operatória ${ }^{22}$.

Os pacientes deste estudo que apresentaram $\mathrm{SpO}_{2}$ menor que $92 \%$ apresentaram valores mais elevados de pressão arterial e de freqüência cardíaca. A hipoxemia aumenta os níveis de catecolaminas circulantes e predispõe à isquemia miocárdica ${ }^{13}$, enquanto a administração de oxigênio reduz a freqüência cardíaca pós-operatória ${ }^{23}$.

A incorporação do oxímetro de pulso na monitorização pós-anestésica permite a utilização seletiva de oxigenioterapia, ou seja, oxigênio é administrado apenas para pacientes que apresentem dessaturação da oxi-hemoglobina. Por exemplo, na SRPA de um hospital geral, oxigênio foi administrado apenas a pacientes que apresentaram $\mathrm{SpO}_{2}$ menor que $94 \%$, o que correspondeu a $37 \%$ dos pacientes estudados, resultando em substancial redução dos custos ${ }^{5}$. Em pacientes submetidas a laparoscopias ginecológicas, utilizando o mesmo critério, oxigênio foi administrado a $15 \%$ dos casos ${ }^{17}$. Neste estudo, oxigenioterapia foi administrada a apenas $23,14 \%$ dos pacientes.

Considerando que, em pacientes monitorizados com oxímetro de pulso, a prevalência de episódios hipoxêmicos depende do grau de motivação do pessoal da SRPA em detectá-los 21 , é possível que o reconhecimento de fatores associados à ocorrência de hipoxemia possa ser utilizado para incentivo do pessoal da SRPA, com a finalidade de aumentar a vigilância, monitorizar mais cuidadosamente os pacientes e, desta forma, detectar episódios hipoxêmicos e administrar precocemente o tratamento.

Concluindo, este estudo identificou fatores associados a maiores chances de ocorrência de hipoxemia no período pós-operatório imediato, que podem identificar pacientes que deverão receber monitorização mais intensiva na SRPA. Conclui-se, também, que a monitorização da $\mathrm{SpO}_{2}$, durante o período pós-anestésico imediato permite a utilização seletiva de oxigenioterapia.

\section{Factors Associated to Hypoxemia in the Immediate Postoperative Period}

Getúlio Rodrigues de Oliveira Filho, M.D., Jorge Hamilton Soares Garcia, M.D., Márcia Regina Ghellar, M.D., Marcos Antônio Nicolodi, M.D., Asmir Luiz Boso, M.D., Adilson José Dal Mago, M.D.

\section{INTRODUCTION}

The routine administration of oxygen in the immediate postoperative period is based on studies showing the high incidence of hypoxemia during this period ${ }^{1,2}$, or those observing a lower incidence of hypoxemia among patients receiving oxygen $^{3,4}$. There are, however, evidences that oxygen is not needed in up to $63 \%$ of patients during the immediate postoperative period ${ }^{5,6}$.

There is hypoxemia even with oxygen administration ${ }^{2,7,8}$ and hypoxemic episodes are very often not diagnosed by the PACU staff ${ }^{2}$. Nevertheless, the use of pulse oximetry in the PACU helps identifying hypoxemic patients and selectively recommending oxygen therapy ${ }^{5,9}$.

Several factors, dependent on patients, anesthetic technique and surgery, are associated to more hypoxemia in the immediate postoperative period ${ }^{7,8}$. Such factors may help to identify patients more prone to develop hypoxemia who, should be more closely monitored by the PACU staff for being candidates to the prophylactic and selective use of oxygen therapy.

This study aimed to detect factors dependent on patients, surgeries and anesthesia, associated to the incidence of hypoxemia in the immediate postoperative period.

\section{METHODS}

After the Medical Ethics Committee, Hospital Governador Celso Ramos approval, participated in this study 216 patients of both genders, aged 15 to 87 years, submitted to elective surgeries under general or regional anesthesia, sequentially admitted to the PACU. Patients under oxygen therapy or mechanical ventilation were excluded. Immediately after admission, patients were monitored with pulse oximetry and non-invasive blood pressure. Peripheral oxy-hemoglobin saturation $\left(\mathrm{SpO}_{2}\right)$, heart rate $(\mathrm{HR})$, systolic (SBP) and diastolic (DBP) blood pressure were recorded at admission (M0), and at 5-minute intervals (M5, M10, M15, M20) for 20 minutes. At the same moments patients received a sedation score $(0=$ agitated, 1 = calm, alert, 2 = sleepy and awaking after verbal command, 3 = sleepy, awaking after tactile stimulation and $4=$ commatous), a ventilation score ( $0=$ apnea, $1=$ limited breathing, respiratory obstruction, bronchospasm or HR below $8 \mathrm{bpm}$, and 2 = free breathing), a muscular strength score $(1=$ keeps grip for $\geq 15$ seconds or $0=$ dos not keep grip or keeps it for less than 15 seconds $)$ and a verbal pain score $(0=$ no pain or no answer, $1=$ mild pain, $2=$ moderate pain and $3=$ severe pain). During the study period, patients only received oxygen via nasal catheter $\left(2 \mathrm{~L} \cdot \mathrm{min}^{-1}\right)$ if their $\mathrm{SpO}_{2}$ went below $92 \%$. The following data were collected: age, gender, weight, height, smoking, history of chronic obstructive pulmonary disease (COPD) or diaVol. 51, No 3, Maio - Junho, 2001 
betes, $\mathrm{SpO}_{2}$ at arrival in the operating room, type and duration of anesthesia, surgery site, neuroaxial use of opioids and perioperative drugs and doses. Clinically detected hypoventilation was defined as a ventilation score equals 1. Patients were divided in hypoxemic, if at any time during the observation period they presented $\mathrm{SpO}_{2}$ below $92 \%$ for more than 30 seconds, and normoxemic, if no $\mathrm{SpO}_{2}$ below $92 \%$ without oxygen therapy was seen during the study period.

Demographics, patients distribution according to the anesthetic technique, surgery site, diabetes, COPD and smoking history, ventilation, sedation, pain and muscular strength scores, the incidence of hypoventilation, the frequency of $\mathrm{SpO}_{2}$ below $95 \%$ at arrival in the operating room, age above 55 years and the frequency of utilization of each perioperative drug were compared among patients with or without hypoxemia by Student's $t$ test for independent samples (continuous variables) or chi-square test (categorical variables) for odds ratio and their $95 \%$ confidence limits calculation. Variables with significant differences between subgroups in the Student's $t$ test or presenting significant association with hypoxemia in the chi-square test were used as independent variables in retrograde logistic regression analysis where the presence or not of hypoxemia $\left(\mathrm{SpO}_{2}\right.$ below 92\%) was the dependent variable. Hemodynamic data were compared between hypoxemic and normoxemic patients using the bi-factorial co-variance analysis for repetitive measures being $\mathrm{SpO}_{2}$ the co-variable in every moment, followed by post hoc Spjotvoll-Stolline test. $\mathrm{SpO}_{2}$ was compared by Friedmans, two-tail Wilcoxon (intragroups comparison) and Mann-Whitney (comparison moment by moment between groups) tests.

The acceptable level of significance was $5 \%$.

\section{RESULTS}

Twelve patients receiving oxygen therapy in the PACU for reasons other than those established in our protocol, such as anemia (2 patients), shivering (2 patients), bronchspasm (1 patient), hypotension ( 4 patients) and unknown reason ( 2 patients) were excluded from the study.

Demographics of the remaining 204 patients are shown in table I. From those, 49 (23.14\%) had $\mathrm{SpO}_{2}$ lower than $92 \%$ for more than 30 seconds and received oxygen therapy. Hypoxemic patients were significantly older than normoxemic patients (Table II).

Table I - Demographics and Major Sample Characteristics

\begin{tabular}{|c|c|}
\hline Age $\left(\right.$ years) ${ }^{1}$ & $45.96 \pm 17.91$ \\
\hline Gender $(\mathrm{M} / \mathrm{F})^{2}$ & $118 / 86$ \\
\hline Weight $(\mathrm{kg})^{1}$ & $69.67 \pm 11.80$ \\
\hline Height $(m)^{1}$ & $1.66 \pm 0.09$ \\
\hline ASA $(I-I I-I I I-I V)^{2}$ & $83 / 100 / 20 / 1$ \\
\hline Smoking (present/absent) ${ }^{2}$ & $61 / 143$ \\
\hline COPD (present/absent) ${ }^{2}$ & $5 / 199$ \\
\hline Diabetes (present/absent) ${ }^{2}$ & $11 / 193$ \\
\hline General/regional anesthesia ${ }^{2}$ & $101 / 103$ \\
\hline Superficial/abdominal surgery ${ }^{2}$ & $74 / 130$ \\
\hline
\end{tabular}

$1=$ Values expressed in Mean \pm SD

$2=$ Values expressed as frequencies

Table II - Demographics Data - Comparison Between Hypoxemic and Normoxemic Patients

\begin{tabular}{lcc}
\hline & Normoxemic & Hypoxemic \\
\hline Age $(\text { years })^{1}$ & $42.99 \pm 15.78$ & $55.36 \pm 18.30$ * \\
Gender $(\mathrm{M} / \mathrm{F})^{2}$ & $93 / 92$ & $25 / 24$ \\
${\text { Weight }(\mathrm{kg})^{1}}^{1}$ & $68.86 \pm 11.28$ & $72.25 \pm 13.08$ \\
Height $(\mathrm{m})^{1}$ & $1.66 \pm 0.09$ & $1.67 \pm 0.09$ \\
\hline
\end{tabular}

$1=$ Values expressed by Mean $\pm S D$

$2=$ Values represent the number of patients in each category

${ }^{*}=p<0.05$ between groups

The following variables were statistically significant in the individual analysis by the chi-square test: physical status ASA III, more than 55 years, preoperative $\mathrm{SpO}_{2}$ below $95 \%$, history of COPD and diabetes, deep sedation (patient awakes only when touched or does not awake), inadequate muscular strength (patient does not keep grip for more than 15 seconds), clinically detected hypoventilation, the use of diazepam or the association of fentanyl and diazepam or midazolam for sedation during regional anesthesia, the association of fentanyl and morphine, the use of vecuronium, enflurane, isoflurane and $\mathrm{N}_{2} \mathrm{O}$, general and regional anesthesia (Tables III and IV). The best logistic regression for our study included general anesthesia with enflurane, clinically detected hypoventilation, age

Table III - Variables Significantly Related to Hypoxemia, their Prevalence in Normoxemic and Hypoxemic Patients and Respective Odds Ratio based on Contingency Tables Analysis (Chi-Square)

\begin{tabular}{|c|c|c|c|}
\hline & Normoxemic & Hypoxemic & $\begin{array}{c}\text { Odds Ratio and Respective } \\
95 \% \text { confidence limits }\end{array}$ \\
\hline \multirow[t]{5}{*}{ Physycal status (ASA) III } & 5,86 & 24.48 * & $5.95(2.07 ; 17.41)$ \\
\hline & 18.70 & 53.06 * & $4.91(2.32 ; 10.41)$ \\
\hline & 2.58 & 14.28 * & $6.29(1.55 ; 27.09)$ \\
\hline & 0.64 & 8.16 * & $13.68(1.38 ; 112.30)$ \\
\hline & 2.58 & 18.36 * & $8.49(2.23 ; 34.84)$ \\
\hline General anesthesia & 41.29 & 79.59 * & $5.54(2.44 ; 12.85)$ \\
\hline Deep sedation ${ }^{1}$ & 5.80 & 20.40 & $4.38(1.50 ; 12.82)$ \\
\hline Inadequate muscular strenght ${ }^{2}$ & 10.32 & 36.73 & $5.35(2.28 ; 12.62)$ \\
\hline Hypoventilation & 3.87 & 57.14 * & $31.11(11.30 ; 102.16)$ \\
\hline
\end{tabular}

$1=$ awakens only when touched or does not awake

$2=$ does not hold grip for 15 seconds

${ }^{*}=p<0.05$ between normoxemic and hypoxemic patients

Revista Brasileira de Anestesiologia

Vol. 51, No 3, Maio - Junho, 2001 
Table IV - Perioperative Drugs related to Hypoxemia, their Use (\%) in Normoxemic and Hypoxemic Patients and Respective Odds Ratio Based on Contingency Table Analysis (Chi-Square)

\begin{tabular}{|c|c|c|c|}
\hline & Normoxemic & Hypoxemic & $\begin{array}{c}\text { Odds Ratio } \\
\text { (95\% confidence limit) }\end{array}$ \\
\hline Diazepam $^{1}$ & 16.77 & 4.08 * & $0.21(0.04 ; 0.96)$ \\
\hline Fentanyl + diazepam ${ }^{1}$ & 13.54 & 2.04 * & $0.13(0.01 ; 0.97)$ \\
\hline Fentanyl + midazolam $^{1}$ & 27.09 & 10.20 * & $0.30(0.09 ; 0.87)$ \\
\hline Fentanyl + morphine & 9.67 & 22.44 * & $2.7(1.05 ; 6.88)$ \\
\hline Vecuronium & 16.12 & 30.61 * & $2.29(1.02 ; 5.13)$ \\
\hline Enflurane & 1.93 & 14.28 * & $8.44(1.85 ; 32.38)$ \\
\hline Isoflurane & 33.54 & 51.02 * & $2.15(1.06 ; 4.37)$ \\
\hline $\mathrm{N}_{2} \mathrm{O}^{2}$ & 22.58 & $40.81 *$ & $3.05(1.46 ; 6.34)$ \\
\hline
\end{tabular}

1 = used for sedation only during regional anesthesia

$2=$ used as volatile anesthetics

${ }^{*} p<0.05$ between groups

above 55 years and $\mathrm{SpO}_{2}$ at operating room arrival below $95 \%$ (Table V). With this model, $96.12 \%$ of normoxemic patients and $65.30 \%$ of hypoxemic patients were correctly classified using the 0.5 logit limit.

Table V - Variables Signficantly Related to Postanesthetic Hypoxemia According to the Logistic Regression Model

\begin{tabular}{lc}
\hline Variable & Odds Ratio and Confidence Limits \\
\hline Age $>55$ years & $4.32(1.70 ; 10.95)$ \\
Preoperative $\mathrm{SpO}_{2}<95 \%$ & $7.47(1.50 ; 37.11)$ \\
General anesthesia with enflurane & $14.53(2.54 ; 82.93)$ \\
Clinically detected hypoventilation & $34.82(11.46 ; 105.84)$ \\
\hline
\end{tabular}

There were no statistically significant differences in: weight, gender, body mass index, physical status, smoking history, volume of crystalloids administered during surgery, surgery duration and site, pain scores, the use of thiopental propofol, midazolam, fentanyl, alfentanil, morphine, atracurium, sevoflurane or neuroaxial opioids (Table VI).

Table VI - Factors Not Significantly Related to Postanesthetic Hypoxemia

\begin{tabular}{lcc}
\hline Variable & Normoxemic & Hypoxemic \\
\hline${\text { Weight }(\mathrm{kg})^{1}}^{1}$ & $68.86 \pm 11.28$ & $72.25 \pm 13.08$ \\
BMI $\left(\mathrm{kg} . \mathrm{m}^{-2}\right)^{1}$ & $24.94 \pm 3.98$ & $25.86 \pm 5.02$ \\
Crystalloids (L) $^{1}$ & $1.45 \pm 0.67$ & $1.52 \pm 0.63$ \\
Duration (min) $^{1}$ & $105.77 \pm 47.21$ & $125 \pm 56.43$ \\
Cavitary surgeries $^{2}$ & 32.25 & 48.97 \\
Sevoflurane $^{2}$ & 2.58 & 10.20 \\
Thiopental $^{2}$ & 21.93 & 30.61 \\
Propofol $^{2}$ & 21.29 & 34.69 \\
Midazolam $^{2}$ & 35.48 & 10.20 \\
Fentanyl $^{2}$ & 68.38 & 69.38 \\
Alfentanil $^{2}$ & 7.74 & 14.28 \\
Morphine $^{2}$ & 14.83 & 28.57 \\
Atracurium $^{2}$ & 13.54 & 26.53 \\
Spinal opioid $^{2}$ & 10.96 & 18.36
\end{tabular}

$1=$ Values expressed in Mean \pm SD

$2=$ Values expressed as incidence percentages

${ }^{*} p<0.05$ between groups

192
Systolic blood pressure, heart rate and hemoglobin peripheral saturation are shown in figures 1,2 and 3 .

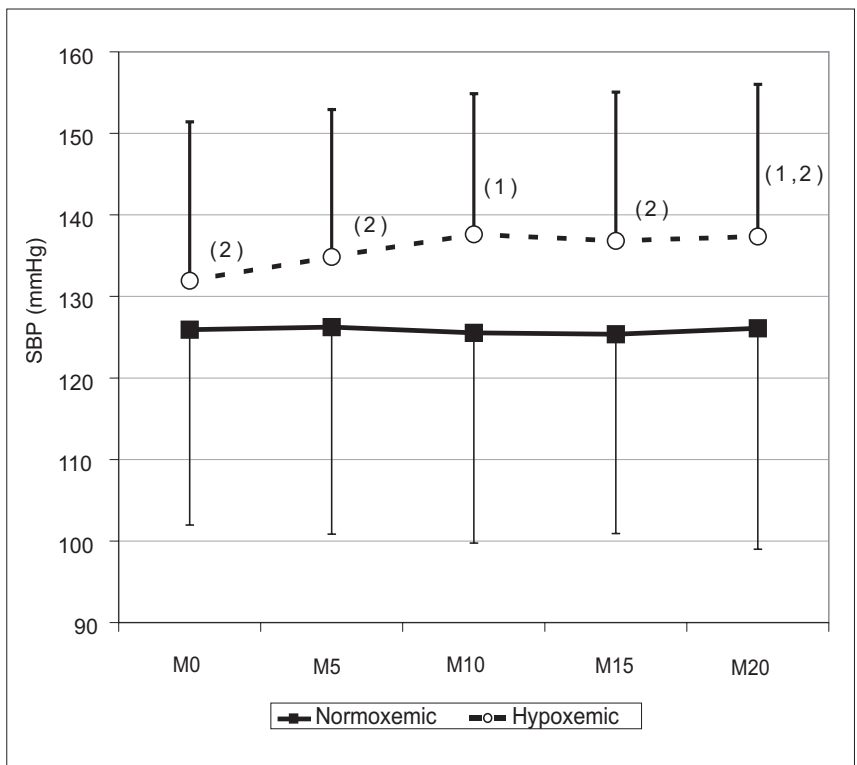

Figure 1 - Systolic Blood Pressure (Mean \pm SD) $1=p<0.05$ as compared to M0 $2=p<0.05$ between groups

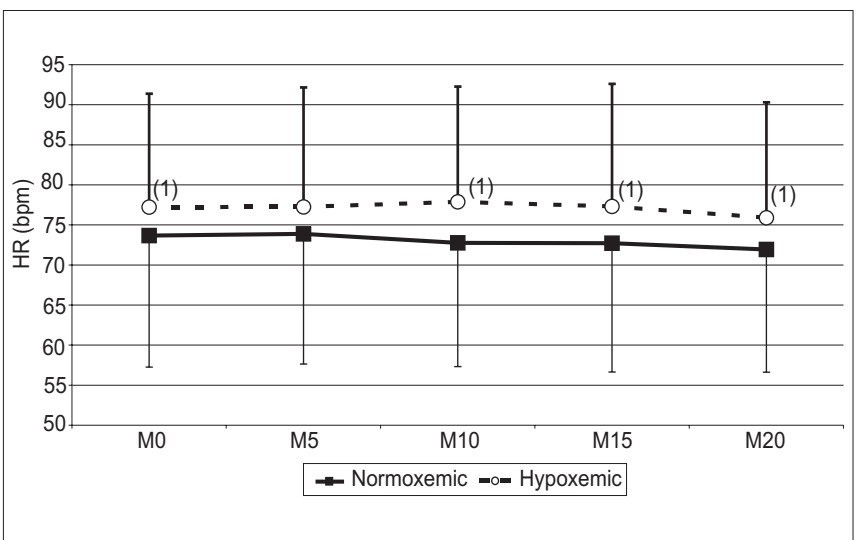

Figure 2 - Heart Rate (Mean \pm SD) $1=p<0.05$ between groups

Revista Brasileira de Anestesiologia Vol. 51, No 3, Maio - Junho, 2001 


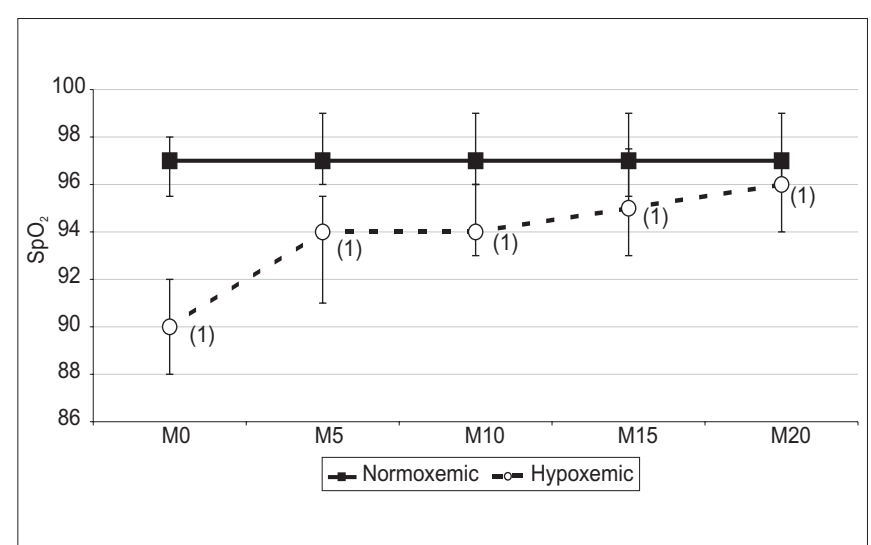

Figure 3 - Hemoglobin Peripheral Saturation (Median, lower and upper quartiles)

$1=p<0.05$ as compared to M0

$p<0.05$ between groups

\section{DISCUSSION}

Postoperative hypoxemia is caused by inadequate ventilation control or by airway patency as a consequence of residual effects of anesthetics and/or neuromuscular blockers and inadequate ventilation/perfusion ratio caused mainly by atelectasis zones in lung-dependent regions ${ }^{10-12}$. Other causes include low cardiac output, anemia, thermogenic shivering, pain and agitation ${ }^{13}$. Most hypoxemic events occur during the 15 minutes following patients' admission to PACU.

Postanesthetic hypoxemia is related to a higher incidence of cardiovascular events during the immediate postoperative period, longer PACU stay and increase in unplanned ICU admissions ${ }^{7}$.

The introduction of pulse oximetry for immediate postanesthetic period monitoring has contributed for the improved identification of hypoxemia and cardiac events such as bradycardia, for decreasing myocardial ischemia, for the use of higher oxygen flows and for a more frequent use of oxygen therapy after PACU discharge, however without changing postoperative mortality or complications rate ${ }^{9}$.

Hypoxemia is defined as oxy-hemoglobin arterial saturation $\left(\mathrm{SaO}_{2}\right)$ below $90 \%$, corresponding to a $\mathrm{PaO}_{2}$ of approximately $60 \mathrm{mmHg}$. Such saturation is found in the inclined portion of the hemoglobin dissociation curve, so that minor $\mathrm{SaO}_{2}$ variations result in marked $\mathrm{PaO}_{2}$ decreases ${ }^{13}$. However, $\mathrm{SpO}_{2}$ overestimates $\mathrm{SaO}_{2}$ in 2 to $5 \%{ }^{14,15}$ justifying lower $\mathrm{SpO}_{2}$ limits between $92 \%$ and $96 \%$ used in several studies ${ }^{5,8,16,17}$.

In studies using $90 \%$ as $\mathrm{SpO}_{2}$ limit, hypoxemia was seen in $0.9 \%{ }^{7}, 4 \%{ }^{6}, 8.24 \%{ }^{18}, 20 \%{ }^{4}, 55 \%{ }^{2}$ and $80 \%{ }^{1}$ of patients. $\mathrm{SpO}_{2}$ limit of $94 \%$ was related to hypoxemia in $15 \%{ }^{17}$ and $37 \%$ ${ }^{5}$ of patients. With the same $\mathrm{SpO}_{2}$ limit, patients under general anesthesia and breathing room air in the PACU had more hypoxemia $(44.7 \%)$ than patients under regional anesthesia $(24 \%)$ or those submitted to general anesthesia and receiving postoperative oxygen therapy $(26.1 \%)^{3}$. In studies with $92 \%$ as $\mathrm{SpO}_{2}$ limit, the incidence of hypoxemia has been $7 \%$
${ }^{16}$ and $15 \%^{8}$, while in our study, hypoxemia was seen in $23.14 \%$ of patients.

In previous studies, variables associated to higher probabilities of immediate postanesthetic hypoxemia were: males, diabetes mellitus, urgency surgeries, postanesthetic medication with opioids and sedatives, fentanyl in doses above 2 $\mu \mathrm{g} \cdot \mathrm{kg}^{-1} \cdot \mathrm{h}^{-1}$, association of fentanyl and morphine, thiopental as compared to propofol ${ }^{7}$, obesity ${ }^{1,7,8,18}$, more than 60 years of age ${ }^{2,7,8,17,18}$, anesthesia for more than 4 hours ${ }^{2,7}$, more than $1500 \mathrm{ml}$ crystalloid infusion ${ }^{8}$, physical status (ASA) ${ }^{8}$ and general anesthesia ${ }^{8}$.

In our study, the isolated analysis of variables has shown a significant association with immediate postoperative hypoxemia of: physical status ASA III, more than 55 years of age, history of COPD or diabetes mellitus, preoperative $\mathrm{SpO}_{2}$ below $95 \%$, general anesthesia, association of perioperative fentanyl with postoperative morphine, use of vecuronium, enflurane, isoflurane and $\mathrm{N}_{2} \mathrm{O}$, deep postanesthetic sedation, inability of keeping grip for 15 seconds and clinical signs of inadequate ventilation.

On the other hand, regional anesthesia, followed or not by perioperative sedation with diazepam and associations of fentanyl and diazepam or midazolam, was related to significantly lower chances of immediate postanesthetic hypoxemia.

The isolated analysis of variables has the disadvantage of not taking into consideration the effect of other factors which may influence possible associations between each variable and a certain event. On the other hand, logistic regression has the advantage of controlling all factors involved in the model by calculating the influence of each variable on the dicotome dependent variable. That is why, after identifying variables potentially related to postoperative hypoxemia by isolated analysis, logistic regression technique was applied and has identified as independent indicators of postoperative hypoxemia only: more than 55 years of age, preoperative $\mathrm{SpO}_{2}$ below $95 \%$, general anesthesia with enflurane and clinically detectable hypoventilation. The remaining variables, significant in isolated analysis, have not contributed to the logistic model and were rejected.

Elderly patients have a decrease in respiratory reserve due to factors such as decrease in nervous breathing control, especially manifested by apnea during sleep, mechanical changes in the chest wall (increase in dorsal syphosis, costal cartilage calcifications and decrease in respiratory muscular mass) and changes in pulmonary volumes and capacities (decrease in maximum respiratory, total and vital pulmonary capacities and increase in airway closing volume $)^{19}$. In addition, elderly patients are more sensitive to depressing effects of inhalational anesthetics, sedatives and opioids. For those reasons, they are more exposed to postoperative hypoxemia as shown by this and other studies $2,7,8,16,18$.

In healthy volunteers, $\mathrm{SpO}_{2}$ below $94 \%$ was seen in just $13.3 \%{ }^{3}$. It is possible that patients with preoperative $\mathrm{SpO}_{2}$ below $95 \%$ have some ventilation abnormality, such as hypoventilation caused, for example, by preanesthetic medication, or abnormality in lungs ventilation/perfusion ratio. 
Patients submitted to general anesthesia have higher chances of developing postoperative hypoxemia ${ }^{3,8}$. In our study, however, only general anesthesia with enflurane was significantly related to hypoxemia in the logistic model, although general anesthesia, isoflurane and $\mathrm{N}_{2} \mathrm{O}$ being significant in isolated analyses. Enflurane is a potent ventilation depressant and causes more hypoxemia in the immediate and late postoperative period ${ }^{20}$. Although not measuring its expired gas concentration, it is possible that the residual effect of enflurane could contribute for immediate postanesthetic hypoxemia.

Clinically detected hypoventilation was the factor more strongly related to hypoxemia. Parameters to define hypoventilation were respiratory rate, diaphragmatic movements inspection, and detection of ventilation abnormalities, such as high respiratory obstruction and bronchospasm. This way, this study confirms the value of clinical evaluation in detecting risk factors for early postoperative hypoxemia.

Similarly to other studies ${ }^{3,8}$, regional anesthesia was related to lower chances of postoperative hypoxemia, even with sedation with diazepam or midazolam, associated or not to fentanyl.

Among the variables studied, weight, gender, body mass index, smoking history, volume of crystalloids administered during surgery, surgery duration and site, the use of thiopental, propofol, midazolam, fentanyl, alfentanil, morphine, atracurium, sevoflurane or neuroaxial opioids, were not significant both in isolated analysis and logistic regression. Hypoxemic patients did not differ from normoxemic patients, except in anesthesia duration. In a previous study ${ }^{2,7}$, anesthesia duration was only a hypoxemia indicator when longer than 4 hours. It is possible that this study failed to identify procedure duration as a factor significantly related to postoperative hypoxemia because mean procedure duration was lower than 4 hours. Smoking was a significant indicator in one study ${ }^{2}$, while in others ${ }^{6,17}$, as well as in our study, there was no significance. The use of morphine for postoperative analgesia was not significantly related to hypoxemia, in line with other authors' findings ${ }^{7}$. So, the risk for hypoxemia does not justify limiting the use of morphine for postoperative analgesia. Cavitary surgeries were no more related to hypoxemia than superficial surgeries, in line with other authors' findings ${ }^{2,7}$. In one study, however, cavitary surgeries were related to higher percentages of postoperative hypoxemia ${ }^{22}$.

In our study, patients with $\mathrm{SpO}_{2}$ below $92 \%$ had higher blood pressure and heart rate values. Hypoxemia increases circulating catecholamine levels and predisposes to myocardial ischemia ${ }^{13}$, while oxygen administration decreases postoperative heart rate ${ }^{23}$.

The use of pulse oximetry for postanesthetic monitoring allows for a selective use of oxygen therapy, that is, oxygen is administered only for patients with oxy-hemoglobin desaturation. For example, in the PACU of a general hospital, oxygen was administered only in patients with $\mathrm{SpO}_{2}$ below $94 \%$, corresponding to $37 \%$ of patients and resulting in substantial cost reductions ${ }^{5}$. In patients submitted to gynecological laparoscopies, using the same criteria, oxygen was administered in $15 \%$ of cases ${ }^{17}$. In our study, oxygen therapy was administered in only $23.14 \%$ of patients. Considering that in patients monitored with pulse oximetry the incidence of hypoxemia depends on the level of motivation of PACU staff to detect it ${ }^{21}$, it is possible that the identification of factors associated to hypoxemia could be used to encourage PACU staff aiming at improving surveillance and patients monitoring, thus detecting hypoxemic episodes and early treating them.

In conclusion, our study has identified factors related to higher chances of immediate postoperative hypoxemia, which may identify patients candidate to a more intensive monitoring in the PACU. Another conclusion is that $\mathrm{SpO}_{2}$ monitoring in the immediate postanesthetic period allows for the selective use of oxygen therapy.

\section{REFERÊNCIAS - REFERENCES}

01. Brown LT, Purcell GJ, Traugott FM - Hypoxaemia during postoperative recovery using continuous pulse oximetry. Anaesth Intensive Care, 1990;18:509-516.

02. Moller JT, Wittrup M, Johansen SH - Hypoxemia in the postanesthesia care unit: an observer study. Anesthesiology, 1990;73:890-895.

03. Smith DC, Canning JJ, Crul JF - Pulse oximetry in the recovery room. Anaesthesia, 1989;44:345-348.

04. Hardeman JH, Sabol SR, Goldwasser MS - Incidence of hypoxemia in the postanesthetic recovery room in patients having undergone intravenous sedation for outpatient oral surgery. J Oral Maxillofac Surg, 1990;48:942-944.

05. DiBenedetto RJ, Graves SA, Gravenstein N et al - Pulse oximetry monitoring can change routine oxygen supplementation practices in the postanesthesia care unit. Anesth Analg, 1994;78:365-368.

06. Gift AG, Stanik J, Kapernick JBS et al - Oxygen saturation in postoperative patients at low risk for hypoxemia: is oxygen therapy needed? Anesth Analg, 1995;80:368-372.

07. Rose DK, Cohen MM, Wigglesworth DF et al - Critical respiratory events in the postanesthesia care unit. Patient, surgical, and anesthetic factors. Anesthesiology, 1994;81:410-418.

08. Russell GB, Graybeal JM - Hypoxemic episodes of patients in a postanesthesia care unit. Chest, 1993;104:899-903.

09. Moller JT, Johannessen NW, Espersen K et al - Randomized evaluation of pulse oximetry in 20,802 patients: II. Perioperative events and postoperative complications. Anesthesiology, 1993; $78: 445-453$.

10. Rothen HU, Sporre B, Engberg G et al - Airway closure, atelectasis and gas exchange during general anesthesia. Br J Anaesth, 1998;81:681-686.

11. Morton CP, Drummond GB - Change in chest wall dimensions on induction of anesthesia: a reappraisal. Br J Anaesth, 1994;73: 135-139.

12. Fairley HB - Oxygen therapy for surgical patients. Am Rev Respir Dis, 1980;122:37-44.

13. Powell JF, Menon DK, Jones JG - The effects of hypoxaemia and recommendations for postoperative oxygen therapy. Anaesthesia, 1996;51:769-772.

14. Seguin P, Le Rouzo A, Tanguy M et al - Evidence for the need of bedside accuracy of pulse oximetry in an intensive care unit. Crit Care Med, 2000;28:703-706.

15. Jensen LA, Onyskiw JE, Prasad NGN - Meta-analysis of arterial oxygen saturation monitoring by pulse oximetry in adults. Heart Lung, 1998;27:387-408.

Revista Brasileira de Anestesiologia Vol. 51, No 3, Maio - Junho, 2001 
16. Murray RS, Raemer DB, Morris RW - Supplemental oxygen after ambulatory surgical procedures. Anesth Analg, 1988;67: 967-970.

17. Vegfors M, Cederholm I, Lennmarken $C$ et al - Should oxygen be administered after laparoscopy in healthy patients? Acta Anaesthesiol Scand, 1988;32:350-352.

18. George JM, Nair L, Dhara SS - Postoperative hypoxaemia during transport and in the recovery area. Ann Acad Med Singapore, 1995;24:807-811.

19. Hedenstierna G, Lofstrom J - Effect of anesthesia on respiratory function after major lower extremity surgery. A comparison between bupivacaine spinal analgesia with low-dose morphine and general anesthesia. Acta Anaesthesiol Scand, 1985;29:55-60.

20. Hoshi K, Shima T, Andoh K et al - Changes in arterial oxygen tension during and after enflurane or halothane anesthesia as well as epidural analgesia. Masui, 1990;39:910-914.

21. Rheineck-Leyssius AT, Kalkman CJ, Trouwborst A - Influence of motivation of care providers on the incidence of postoperative hypoxaemia in the recovery room. Br J Anaesth, 1996;77: 453-457.

22. Xue FS, Zhang GS, Liao X et al - The influence of surgical sites on early postoperative hypoxemia in adults undergoing elective surgery. Anesth Analg, 1999;88:213-219.

23. Rosenberg-Adamsen S, Lie C, Bernhard A et al - Effect of oxygen treatment on heart rate after abdominal surgery. Anesthesiology, 1999;90:380-384.

\section{RESUMEN}

Oliveira Filho GR, Garcia JHS, Ghellar MR, Nicolodi MA, Boso AL, Dal Mago AJ - Factores Asociados con la Ocurrencia de Hipoxemia en el Período Pós-Anestésico Inmediato

Justificativa y Objetivos - La hipoxemia pós-anestésica es un evento respiratorio crítico que aumenta la morbidad pós-operatoria. Este estudio busca identificar factores relacionados a la ocurrencia de hipoxemia en el período pós-operatorio inmediato.

Método - Fueron incluidos 204 pacientes admitidos a la sala de recuperación pós-anestésica respirando aire ambiente. La saturación periférica de la oxi-hemoglobina $\left(\mathrm{SpO}_{2}\right)$, las presiones arteriales sistólica (PAS) y diastólica (PAD) y la frecuencia cardíaca (FC) fueron medidas a intervalos de 5 minutos desde la admisión del paciente hasta 20 minutos después. Escores de sedación, de dolor y de adecuación de la ventilación fueron atribuidos en los mismos momentos. $\mathrm{SpO}_{2}$ menor que $92 \%$ fue definida como hipoxemia e indicación para oxigenioterapia. Fueron registrados: edad, sexo, peso, altura, historia de fumo, de DPOC y de diabetes melito, $\mathrm{SpO}_{2}$ en la llegada a la sala de cirugía, tipo de anestesia, región operada, duración da anestesia, uso de opioides neuro-axiales y drogas utilizadas en el período peri-operatorio y respectivas dosis. La asociación de estos factores con hipoxemia fue definida por regresión logística.

Resultados - Cuarenta y nueve pacientes (24,01\%) presentaron $\mathrm{SpO}_{2}$ menor que $92 \%$ durante el período de observación. Fueron indicadores de hipoxemia [relación de veces (limites de $95 \%$ de confianza)]: edad mayor que 55 años $[4,32(1,70 ; 10,95)], \mathrm{SpO}_{2}$ pré-operatoria menor que $95 \%[7,47$ $(1,50 ; 37,11)]$, anestesia general con enflurano $[14,53$ $(2,54 ; 82,93)]$, hipoventilación detectada clínicamente [34,82 $(11,46 ; 105,84)]$. La PAS y la FC fueron significativamente mas elevadas en los pacientes hipoxémicos.

Conclusiones - Existen factores significativamente asociados a la ocurrencia de hipoxemia pós-operatoria, en cuanto el uso del oxímetro de pulso permite la utilización selectiva de oxigenioterapia en el período pós-anestésico inmediato. 